\title{
Autoantibody IgG Protects Bone Erosion and Destruction in SLE Arthritis
}

\author{
Guo Min Deng* \\ Department of Rheumatology, Huazhong University of Science and Technology, China
}

*Corresponding author: Guo Min Deng, Department of Rheumatology, Union Hospital, Tongji Medical College, Huazhong University of Science and Technology, Wuhan, 430022, China.

To Cite This Article: Guo Min Deng. Autoantibody IgG Protects Bone Erosion and Destruction in SLE Arthritis. 2020 - 11(1). AJBSR.MS.ID.001605. DOI: 10.34297/AJBSR.2020.11.001605.

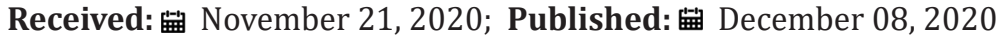

\section{Opinion}

Systemic lupus erythematosus (SLE) is a chronic autoimmune disease characterized by high levels of autoantibodies and multiorgan tissue damage [1]. Arthritis is common in patients with SLE [2]. Bone erosion is a remarkable feature in arthritis such as rheumatoid arthritis (RA), but they are usually absent in arthritis of SLE [3]. Thus, this is particularly striking for clinical doctors because synovial biopsies from SLE patients show similar synovial inflammation to those in RA [4]. Tissue deposited lupus IgG displays a key role in initiating inflammation in organ tissue including kidney, brain, skin and liver [5-7]. It remains unknown whether and how joint deposited lupus IgG contributes to arthritis without bone erosions in SLE. The recent published paper presents data that lupus IgG deposition causes arthritis but inhibits bone destruction through competitive occupation of Fc $\gamma$ RI and reduced RANKL signaling [8], intraarticular injection of lupus IgG triggers arthritis, but does not results in bone destruction, tissue deposition of lupus IgG, monocytes are required for the development of this arthritis [8]. Lupus IgG blocks RANKL-induced monocyte differentiation into osteoclasts that contribute to bone erosion, Fc $\gamma$ RI exerts a critical role in pathogenesis of lupus arthritis lacking bone erosion [8].

In lupus MRL/lpr mice, there is IgG deposition and synovial inflammation in absence of bone erosions in joints [8]. In normal mice, intraarticular injection of lupus IgG can trigger synovial inflammation but no bone destruction [8]. Arthritis occurred 3 hours after injection, peaked after 3 days, lasted for at least 14 days and the severity of the arthritis was dose dependent. In mouse model of arthritis induced by intraarticular injection of lupus IgG, the severity of arthritis was significantly reduced in mice with monocyte depletion but not affected in mice with lymphocyte deficiency and mice with neutrophil depletion [8]. These results indicate that lupus IgG deposited in the joint can trigger arthritis by activating monocytes/macrophages [8].

Because monocytes/macrophages can differentiate into osteoclasts in the presence of RANKL [9], Fc $\gamma$ R is not only receptor for IgG but also is required for RANKL-induced osteoclast genesis $[10,11]$, the role of lupus IgG on RANKL-induced monocytes differentiation into osteoclast was determined. The result demonstrates that lupus IgG directly inhibited RANKL-induced monocytes differentiation into osteoclast [8]. Deficiency of Fc $\gamma$ RIIB or Fc $\gamma$ RIII did not affect inhibitory effect of lupus IgG on RANKLinduced monocyte differentiation into osteoclast. Data from flow cytometry demonstrates that lupus IgG significantly decreased surface level of Fc $\gamma$ RI but not Fc $\gamma$ RII and Fc $\gamma$ RIII on monocytes. Data demonstrate that RANKL significantly reduces surface level of Fc $\gamma$ RI but not Fc $\gamma$ RII and Fc $\gamma$ RIII on monocytes [8]. These results indicate that lupus IgG inhibits RANKL-induced osteoclast genesis through Fc $\gamma$ RI.

Relationship among lupus IgG, RANKL and Fc $\gamma$ RI was investigated. The stronger inhibitory effect on osteoclast genesis was observed in high doses of lupus IgG than lower doses of lupus IgG [8]. Inhibitory effects of lupus IgG on osteoclast genesis was gradually blocked by increasing doses of RANKL suggesting competition between lupus IgG and RANKL. The stronger inhibitory effects of lupus IgG on RANKL-induced osteoclast genesis were shown in cells pretreated for $24 \mathrm{~h}$ when compared to cells treated with both RANKL and IgG at the same time [8]. This data indicates that inhibitory effects of lupus IgG on osteoclast genesis depend on the extent of IgG binding to Fc $\gamma$ RI. The inhibitory effect of lupus 
IgG on osteoclast genesis was converted at 24 hours after RANKL stimulation [8]. It suggests that inhibitory effect of lupus IgG on osteoclast genesis is blocked by the extent of RANKL binding to Fc $\gamma$ RI.

These data suggest that lupus IgG inhibits RANKL-induced osteoclast genesis through competition for Fc $\gamma$ RI binding [8] This study demonstrates that lupus IgG can induce synovitis but inhibit RANKL-induced osteoclast genesis, suggesting that joint deposited lupus IgG can have different effects on joint tissues [8]. Recruitment of lupus IgG to Fc $\gamma$ RI may result in functional deficiency of Fc $\gamma$ RI on the cellular membrane, which is required for RANKL-induced osteoclast genesis [8]. This study enhances the understanding of pathogenesis of non-destructive arthritis in SLE. The competitive occupation of Fc $\gamma$ RI by IgG may develop new therapeutic approaches to prevent bone destruction in autoimmune/inflammatory arthritis.

\section{Acknowledgments}

This study was supported by Research Initiating Fund (GM Deng, 02.03.2018-41) of Union hospital affiliated to Tongji Medical College, Huazhong University of Science and Technology.

\section{Conflict of Interest}

There is no conflict of interest.

\section{References}

1. Tsokos GC (2011) Systemic lupus erythematosus. N Engl J Med 365 2110-2121.
2. Gormezano NW, Silva CA, Aikawa NE, Barros DL, MA da Silva, et al. (2016) Chronic arthritis in systemic lupus erythematosus: distinct features in 336 paediatric and 1830 adult patients. Clin Rheumatol 35(1): 227-231.

3. Martinez JB, Valero JS, Bautista AJ, Restrepo JF, Matteson EL, et al. (2007) Erosive arthropathy: clinical variance in lupus erythematosus and association with anti-CCP case series and review of the literature. Clin Exp Rheumatol 25(1): 47-53.

4. Seibold JR, Wechsler LR, Cammarata RJ (1980) LE cells in intermittent hydrarthrosis. Arthritis Rheum 23(8): 958-959.

5. Liu Z, Davidson A (2012) Taming lupus-a new understanding of pathogenesis is leading to clinical advances. Nat Med. 18(6): 871-882

6. Deng GM, Liu L, Kyttaris VC, Tsokos GC (2010) Lupus serum IgG induces skin inflammation through the TNFR1 signaling pathway. J Immunol 184(12): 7154-7161.

7. Fang X, Zaman MH, Guo X, Ding H, Xie C, et al. (2018) Role of Hepatic Deposited Immunoglobulin G in the Pathogenesis of Liver Damage in Systemic Lupus Erythematosus. Front Immunol 9: 1457

8. Qiao W, Ding H, Zuo Y, Jiang L, Zhou J, et al. (2020) Lupus IgG deposition causes arthritis but inhibits bone destruction through competitive occupation of Fc $\gamma$ RI and reduced RANKL signalling. Clin Transl Immunology 9(9): e1174

9. Deng GM, Zheng L, Chan FK, Lenardo M (2005) Amelioration of inflammatory arthritis by targeting the pre-ligand assembly domain of tumor necrosis factor receptors. Nat Med 11(10): 1066-1072.

10. Takai $\mathrm{T}$ (2002) Roles of Fc receptors in autoimmunity. Nat Rev Immunol 2(8): 580-592.

11. Koga T, Inui M, Inoue K, Kim S, Suematsu A, et al. (2004) Costimulatory signals mediated by the ITAM motif cooperate with RANKL for bone homeostasis. Nature 428(6984): 758-763. 